\title{
Editorial: A Tale of Two Anniversaries: 125 Years of the Physical Review and 25 Years of Physical Review $E$
}

In July 1893, Ernest F. Nichols, then a professor of physics at Colgate University and later president of Dartmouth College and MIT, published an account of fundamental research in spectroscopy entitled "A Study of the Transmission Spectra of Certain Substances in the InfraRed." The paper, which appeared 125 years ago this year was the first ever to be published in The Physical Review [E. F. Nichols, Phys. Rev. 1, 1 (1893)].

The first issue of The Physical Review, covering the months of July and August 1893, was a slender one. Nichols's paper was one of just five articles published in the inaugural issue, which contained a mere 80 printed pages.

How times have changed; fast forward to 2017: In the same period-July and Augustalmost 3,500 original research papers and more than 33,000 pages were published in the Physical Review journals which had by then expanded to a portfolio of 13 peer reviewed general and specialist titles. The remarkable growth in volume and breadth of The Physical Review - publication of which was taken over by the American Physical Society (APS) in 1913 - has tracked the increase in understanding and profile of physics, and has been made possible by the great vision of past leaders of The Physical Review and the APS.

The landscape of scientific publication has changed significantly in the 125 years since E. F. Nichols's inaugural paper graced The Physical Review, but the Physical Review journals have proved equal to the challenge of meeting the constantly evolving requirements of the physics research community. An early innovation was the launch of Reviews of Modern Physics (RMP) in 1929 which fulfilled the demand for a high-quality journal offering authoritative accounts of the current state of the increasingly numerous and diverse fields in physics. Revolutionary at the time, RMP quickly established a reputation as the foremost reviews journal in physics, a position it has maintained for almost nine decades.

Another challenge came with the research boom of the post-war period which brought with it a growing need for swift publication of high-profile results. This led to the creation in 1958 of Physical Review Letters - the world's premier physics letters journal—which for 60 years has published short, high-quality reports of significant and notable results in the full arc of fundamental and interdisciplinary physics research.

Further growth in the number of papers published in The Physical Review led to its division into a number of individual topical journals-Physical Review A-D in 1970 and Physical Review $E$ in 1993. In subsequent years a number of specialized journals focused on individual research fields were added. Other advances and challenges made possible by technology, such as online electronic publishing and most recently the demand for open access publishing, have all been embraced by the Physical Review journals.

Over the 125 years since the publication of Nichols's inaugural paper, the Physical Review portfolio has grown into the largest and most complete suite of physics journals in the world. As of 2017, this comprises 13 peer-reviewed journals — of which three are fully open accesspublishing over 19,000 original research and review articles. Many of these were highlighted in the free APS online commentary journal Physics - just one way in which APS adds value to the high-quality research it publishes.

From Robert Millikan's famous oil drop experiments to the discovery of gravitational waves, many history-making discoveries have been published in the Physical Review journals. To celebrate the $125^{\text {th }}$ anniversary of The Physical Review, throughout 2018 APS will spotlight one paper of great importance per week from the journals that document the tremendous advances in physics as they appeared to our readers.

Choosing these articles, which will be made free to read, was a tremendously difficult task and resulted in much passionate debate among the editors. Many other worthy papers could easily have been included and even some papers cited in the awarding of a Nobel Prize did not make the final cut. 
All selected papers are displayed on a timeline at the website journals.aps.org/125years along with landmark events in the history of The Physical Review. Throughout 2018 we will also highlight these papers on social media (\#PhysRev125) and we hope and anticipate that this selection will spur lively conversations within the physics community. We encourage you to join the discussions either online or in person with the editors at one of the many meetings they plan to attend around the world. A list of these meetings can be found on the individual journal websites. Please take these opportunities to meet the editors who are always open for suggestions, criticism, as well as praise.

In addition to the $125^{\text {th }}$ anniversary of Physical Review, 2018 also marks the $25^{\text {th }}$ anniversary of Physical Review E. This landmark will be celebrated by spotlighting one article-25 in total-from each year of publication. Each of these seminal articles in statistical, nonlinear, biological, or soft matter physics will be marked as a PRE Milestone and will be featured on the journal's website.

While reminding us of our history, anniversaries also provide a valuable impetus to look to the future. In their first 125 years the Physical Review journals have made significant contributions to the dissemination of science. As physics continues to grow and form synergies with other fields of science, the Physical Review journals will again need to adapt to the changing needs of this broad and expanding community to remain current, compelling, and relevant.

The scientific publishing landscape itself will surely continue to evolve at increasing speed in the years to come. Input from all our stakeholders-authors, referees and readers-will as always be crucial to ensuring that the Physical Review journals anticipate and prepare for these changes so they continue to meet the needs of the community for the next 125 years and beyond.

Published 2 January 2018

Matthew Salter

Publisher

Michael Thoennessen

Editor in Chief

DOI: 10.1103/PhysRevD.97.020001 